\title{
Yes-Associated Protein is Involved in Myocardial Fibrosis in Rats with Diabetic Cardiomyopathy
}

\author{
Maomao $\mathrm{Hu}^{1,2, *}$ \\ Han Wang ${ }^{1-3, *}$ \\ Shengnan $\mathrm{Li}^{1,2, *}$ \\ Feng Yan (iD ${ }^{2}$ \\ Changning $\mathrm{Fu}^{1,2}$ \\ Lei $\mathrm{Li}^{1,2}$ \\ Yalin $\mathrm{Yu}^{1,2,4}$ \\ Jie Xiong ${ }^{1,2}$ \\ Bo Dong ${ }^{1,2,4}$
}

'Department of Cardiology, Shandong Provincial Hospital Affiliated to Shandong University, Jinan, 2500I2, People's Republic of China; ${ }^{2}$ Key Laboratory of Cardiovascular Remodeling and Function Research, Qilu Hospital, Shandong University, Jinan, People's Republic of China; ${ }^{3}$ Department of Cardiology, Shandong Provincial Hospital Affiliated to Shandong First Medical University, Jinan, People's Republic of China; ${ }^{4}$ Department of Cardiology, Shandong Traditional Chinese Medicine University, Jinan,

People's Republic of China

*These authors contributed equally to this work
Correspondence: Bo Dong; Jie Xiong Department of Cardiology, Shandong Provincial Hospital Affiliated to Shandong University, Jinan, Shandong Province, 2500 I2, People's Republic of China Email bodong@sdu.edu.cn; xiongjiedel63@I63.com
Introduction: Recent studies have shown that YAP is closely related to the pathological process of cardiovascular diseases. But the role of YAP in cardiac injury of diabetic cardiomyopathy (DCM) is still unclear.

Methods: Diabetic cardiomyopathy rat model was established and divided into control group, DCM group, LV-SC-shRNA group and LV-YAP-shRNA group. LV-SC-shRNA group and LV-YAP-shRNA group were injected with lentivirus expressing SC-shRNA and YAP-shRNA via tail vein, respectively. Primary rat cardiac fibroblasts (CFs) were stimulated with high concentration of glucose and treated with recombinant lentivirus expressing either SC-shRNA or YAP-shRNA to observe the expression of CTGF and fibronectin, so as to observe the effect of inhibiting YAP on the pathogenesis of DCM.

Results: Compared with control group, high glucose markedly increased YAP mRNA and protein expression in DCM and CFs. Inhibition of YAP decreased myocardial fibrosis and improved cardiac function in the DCM model and decreased the expression of CTGF and fibronectin in CFs. The result suggested that YAP plays a key role in the pathological progression of DCM, and the underlying mechanisms may be associated with TEAD and CTGF.

Discussion: We found that the expression of YAP was increased both in vivo and in vitro, suggesting that YAP is closely related to DCM, and YAP knockdown can reduce myocardial fibrosis in rat model of DCM by reducing the expression of PAI-1, collagen I, collagen III, CTGF and profilin, as well as the expression of CTGF and fibronectin in CFs. This study revealed that YAP plays an important role in the pathological process of diabetic cardiomyopathy, and down-regulation of YAP expression may provide a new therapeutic target for DCM.

Keywords: diabetic cardiomyopathy, yes-associated protein, myocardial fibrosis

\section{Introduction}

It is well known that with the rapid increase in the global prevalence of cardiovascular disease and death in diabetes mellitus (DM), the risk of people with diabetes mellitus also increases significantly. ${ }^{1,2}$ Cardiovascular complications are the leading cause of diabetesrelated morbidity and mortality. ${ }^{2}$ Diabetic cardiomyopathy (DCM) is characterized by left ventricular hypertrophy and reduced diastolic function. ${ }^{3}$ The potential mechanisms behind DCM include damage from reactive oxygen species, oxidative stress, inflammation, and myocardial fibrosis. ${ }^{4-7}$ Study showed that myocardial fibrosis is a important feature of DCM, and accumulation of extracellular matrix proteins, including, collagen I, collagen III, CTGF and fibronectin expression, has been documented. Although multiple potential mechanisms have been studied, the pathological mechanism of DCM remains unclear. 
Yes-associated protein (YAP), a $65 \mathrm{kDa}$ binding partner of Src family kinase c-Yes, is the primary effector molecule in the Hippo pathway, it was first discovered in Drosophila. ${ }^{8} 9$ Dephosphorylation of YAP and TAZ (transcriptional coactivator with PDZ-binding motif) induces the nuclear translocation of these proteins and plays a crucial role in the Hippo pathway, this pathway is composed of a highly conserved core kinase cascade regulated by multiple sources of upstream input and has multiple transcriptional outputs. ${ }^{10}$ Furthermore, YAP and TAZ are transcriptional coregulators that bind primarily to enhancer elements by associating with TEAD factors (TEA domain family members; also known as TEFs), which are sequence-specific DNA-binding proteins, to induce transcription of target genes. ${ }^{11}$ Meanwhile, YAP and TAZ are crucial for a process that converts extracellular mechanical cues into intracellular signaling, and they are known to regulate cell proliferation, survival, polarity, organ size, vascular development and pulmonary parenchymal fibrosis, particularly in development and cancer progression. ${ }^{12}$

Recent studies have shown that YAP is closely related to the pathological process of cardiovascular diseases. ${ }^{11-13}$ Deletion of YAP specifically in cardiac and vascular smooth muscle cells influenced the development of cardiovascular disease in mice. ${ }^{14}$ Moreover, YAP protein expression has been found to be increased in diabetic mouse kidneys. ${ }^{15}$ However, there is no clear elucidation of the role of YAP in diabetic cardiomyopathy.

In view of the relationship between YAP and cardiovascular diseases, we hypothesized that YAP may be involved in the development of DCM. Therefore, we designed and conducted a series of in vitro and in vivo experiments to verify our speculation that YAP may aggravate the fibrosis of DCM and further aggravate the pathological process of DCM.

\section{Materials and Methods}

\section{Recombinant Lentivirus Expressing shRNA Targeting YAP}

Short hairpin RNA (shRNA) fragments corresponding to Yes-associated protein (YAP) were constructed and were introduced into a recombinant lentivirus by Genechem to form a lentivirus-coated YAP shRNA fragment. According to Wang et al. ${ }^{16}$

\section{Animal Model}

Sixty male 8-week-old Wistar rats $(200 \pm 12$ g) were obtained from Shandong University Animal Center. All animals were maintained in a $12 \mathrm{~h}$ light and $12 \mathrm{~h}$ dark cycle at an ambient temperature of $25 \pm 2^{\circ} \mathrm{C}$ and $50 \pm 10 \%$ humidity with free access to standard rodent chow and water. After a 1-week acclimatization period, the animals were randomly divided into the following four groups: control group $(\mathrm{n}=15)$, DCM group $(\mathrm{n}=15), \mathrm{DCM}$ +Scramble-shRNA group (LV-SC-shRNA, $\mathrm{n}=15$ ), and DCM+YAP-shRNA group (LV-YAP-shRNA, n=15). After fasting for over $12 \mathrm{~h}$, diabetic rats were injected with single and high doses of streptozotocin $(65 \mathrm{mg} / \mathrm{kg})$ to construct diabetes model. One week after administration of streptozotocin, Wistar rats with plasma glucose levels $>$ $11.1 \mathrm{mmol} / \mathrm{L}$ and typical clinical symptoms of polydipsia, polyphagia and polyuria were considered diabetic rat models. Twelve weeks after administration of streptozotocin, rats in the DCM+Scramble-shRNA group and the DCM +YAP-shRNA group were injected via the tail vein with $1 \times 10^{6}$ PFU of either the Scramble-shRNA or YAP-shRNA recombinant lentivirus (dissolved in $100 \mu \mathrm{L}$ normal saline) per rat.

Four weeks after the recombinant lentivirus injection, all rats, including normal rats and diabetic rats, were euthanized with an intraperitoneal injection of $10 \%$ chloral hydrate $(300 \mathrm{mg} / \mathrm{kg})$ and then were sacrificed. The hearts were collected for further experiments.

\section{Echocardiography Analysis}

Before rats were sacrificed under anesthesia with intraperitoneal injection of $10 \%$ chloral hydrate $(300 \mathrm{mg} / \mathrm{kg})$, echocardiography was performed with a VEVO 770 echocardiography system equipped with a $25 \mathrm{MHz}$ transducer (Visual Sonics, Toronto, Canada). M-mode signals were collected, and data on the following measures of left ventricular functions were collected: left ventricular ejection fraction (LVEF), left ventricular end-diastolic diameter (LVEDD), left ventricular end-systolic diameter (LVESD), and the ratio of flow Doppler $\mathrm{E}$ wave to A wave amplitude (E/A).

\section{Histopathology and Immunohistochemistry}

The myocardial tissues were fixed with $4 \%$ paraformaldehyde for more than $24 \mathrm{~h}$, flushed with water for at least 12 $\mathrm{h}$, dehydrated with an ethanol gradient and embedded in paraffin. Then, $4.5-\mu \mathrm{m}$-thick tissue sections were cut for use in different experiments. Immunohistochemical staining was performed with $4.5-\mu \mathrm{m}$-thick tissue sections 
obtained from paraffin-embedded myocardial tissues from the four groups. The sections were first deparaffinized and rehydrated, and then antigen retrieval was performed at a temperature over $95^{\circ} \mathrm{C}$ for 15 minutes. After antigen retrieval, the sections were cooled to room temperature and blocked with goat serum for 15 minutes at $37^{\circ} \mathrm{C}$; then, the sections were incubated overnight at $4^{\circ} \mathrm{C}$ with primary antibodies against rat YAP (1:200, Cell Signaling Technology, Danvers MA, USA), collagen I (1:200, ab34710, Abcam, UK), collagen III (1:100, ab7778, Abcam, UK), CTGF (1:100, DF7091, Affinity, USA), PAI-1 (1:100, AF5176, Affinity, USA,) and profilin (1:200, Abcam, Cambridge, MA). Negative controls were treated without a primary antibody. After rewarming the sections in an incubator at $37^{\circ} \mathrm{C}$ for 30 minutes, they were incubated with a secondary antibody and were subjected to DAB staining. Secondary antibody incubation and DAB color development were performed according to the manufacturer's instructions (ZSGB-Bio, China, ZB-2301). All histopathological sections were photographed under a confocal FV 1000 SPD laser scanning microscope (Olympus, Japan) and were analyzed by a customized imaging analysis system (Image-Pro Plus 6.0, Media Cybernetics, Rockville, MD, USA).

\section{Cell Culture}

Primary rat neonatal Cardiac fibroblasts (CFs) were isolated from 1- to 3-day-old Wistar rats. CFs between passages 2 and 3 were seeded in the six-well cell plates and were used for further experiments. The cells were stimulated with high glucose $(25 \mathrm{mM})$ in a time-dependent manner at $0 \mathrm{~h}, 6 \mathrm{~h}, 12 \mathrm{~h}, 24 \mathrm{~h}$ and $48 \mathrm{~h}$. Cardiac fibroblasts (CFs) were stimulated in $25 \mathrm{mM}(\mathrm{HG})$ glucose medium for $6,12,24,48$ and 72 hours.

RT-PCR and Western blot were used to evaluate the expression of YAP in CFs to identify the optimal conditions.

To evaluate the effects of YAP-shRNA-mediated YAP silencing on high glucose-induced injury of neonatal CFs, cells were transfected with LV-YAP-shRNA or LVScramble-shRNA. After transfection, the cells were incubated in medium containing $25 \mathrm{mM}$ glucose for $48 \mathrm{~h}$. All cell experiments were repeated three times.

Primary rat neonatal Cardiac fibroblasts (CFs) were isolated from 1- to 3-day-old Wistar rats. CFs between passages 2 and 3 were seeded in the six-well cell plates and were used for further experiments. The cells were stimulated with high glucose $(25 \mathrm{mM})$ in a time-dependent manner at $0 \mathrm{~h}, 6 \mathrm{~h}, 12$ h, $24 \mathrm{~h}$ and $48 \mathrm{~h}$. RT-PCR and Western blotting were used to evaluate the expression of YAP in CFs to identify the optimal conditions.

To evaluate the effects of YAP-shRNA-mediated YAP silencing on high glucose-induced injury of neonatal CFs, cells were transfected with LV-YAP-shRNA or LVScramble-shRNA. After transfection, the cells were incubated in medium containing $25 \mathrm{mM}$ glucose for $48 \mathrm{~h}$. All cell experiments were repeated three times.

\section{Western Blot Analysis}

Total protein from CFs and frozen stored cardiac tissue extracts were collected following lysis in RIPA lysis buffer containing protease inhibitor cocktail, then the protein levels were quantified. Protein $(30 \mu \mathrm{g} /$ lane $)$ was separated by SDS-PAGE and was transferred to polyvinylidene difluoride membranes. After blocking nonspecific proteins in Tris-buffered saline containing 5\% skim milk for $1 \mathrm{~h}$, the membranes were incubated overnight at $4{ }^{\circ} \mathrm{C}$ with the following primary antibodies: anti-rat YAP (1:1000, Cell Signaling Technology, Danvers MA, USA), fibronectin (1:1000, Abcam, Cambridge, MA) and CTGF (1:800, DF7091, Affinity, USA,). Then, all membranes were incubated with a horseradish peroxidase-conjugated secondary antibody (1:5000, ZSGB-bio, China, ZB-2301) to bind to the specific antigen-antibody complex for $2 \mathrm{~h}$ at room temperature. The immunoreactive bands were visualized by an enhanced chemiluminescence reagent, and the intensity of bands was quantitated by ImageJ (National Institutes of Health, Bethesda, MD).

\section{Real-Time PCR}

Total RNA was purified from the myocardial tissues and cardiac fibroblasts by using TRIzol Reagent (Invitrogen, USA) according to the manufacturer's instructions. The quantity of total RNA was determined by OD260 measurements. Total RNA was reverse-transcribed with Super ScriptIII (Takara, Japan) according to the manufacturer's protocol. Then, cDNA was amplified using SYBR Green (Takara, Japan). Relative gene expression was calculated using the $2^{-\Delta \Delta \mathrm{CT}}$ method.

\section{Coimmunoprecipitation (Co-IP) Assays}

Cardiac fibroblasts were lysed with RIPA buffer, and the resulting cell suspension was centrifuged at $14,000 \mathrm{~g}$ at $4^{\circ}$ $\mathrm{C}$ for 5 minutes, and then the supernatant was collected. Protein G-sepharose beads were added to the cells, and the resulting solution was shaken at $4^{\circ} \mathrm{C}$ for $2 \mathrm{~h}$. The mixture 
was centrifuged at $2500 \mathrm{~g}$ at $4^{\circ} \mathrm{C}$ for 5 minutes, and then the supernatant was collected. Rat anti-YAP was added to the supernatant, and they were subjected to shaking at $4^{\circ} \mathrm{C}$ overnight. Protein A-sepharose beads were added to the supernatant and were subjected to shaking at $4^{\circ} \mathrm{C}$ for $4 \mathrm{~h}$. The bead-antibody complex was washed five times with chilled PBS and then was boiled for 10 minutes to obtain the protein supernatant. The protein supernatant was used for Western blot analysis to verify the binding between YAP and TEAD.

\section{Chromatin Immunoprecipitation (Ch-IP)}

\section{Assays}

Ch-IP was performed using a Simple Ch-IP Plus Enzymatic Chromatin IP Kit (Cell Signaling Technology, Danvers, USA) following the protocol provided by the manufacturer. Briefly, CFs were cross-linked, lysed, and sonicated to generate DNA fragments of 150 to $900 \mathrm{bp}$. The sheared chromatin was incubated with a YAP antibody (Cell Signaling Technology, Danvers MA, USA) and protein G magnetic beads. IgG was employed as the negative control. Ch-IP DNA was amplified by using primers for the CTGF promoter and analyzed by qPCR and sequencing. Five primers were designed based on different segments of the CTGF promoter as follows: 1 forward: AAAGCGTGAGGCCAAGTTGC; and reverse: AG TGCAGGAAGGATTTCCAACAT; 2 forward: CCATGGA TGGACTGAAAAAGTTGT; and reverse: GTGGCTTC CTCTCATAGTTGGT; 3 forward: AACAAACTCCAAC AGCATCGT; and reverse: TCAGGGAACATAG GGTGGGA; 4 forward: CCCGTGGTATCTGCCTCTTC; and reverse: CTTCGCACCCTTCCTGATGA; 5 forward: TG CCAGCTTTTTCAGACGGA; and reverse: TGGCTTTTATACGCTCCGGG.

\section{Cell Culture}

Primary rat neonatal Cardiac fibroblasts (CFs) were isolated from 1- to 3-day-old Wistar rats. CFs between passages 2 and 3 were seeded in the six-well cell plates and were used for further experiments. The cells were stimulated with high glucose $(25 \mathrm{mM})$ in a time-dependent manner at $0 \mathrm{~h}, 6 \mathrm{~h}, 12 \mathrm{~h}, 24 \mathrm{~h}$ and $48 \mathrm{~h}$. RT-PCR and Western blotting were used to evaluate the expression of YAP in CFs to identify the optimal conditions.

To evaluate the effects of YAP-shRNA-mediated YAP silencing on high glucose-induced injury of neonatal CFs, cells were transfected with LV-YAP-shRNA or LVScramble-shRNA. After transfection, the cells were incubated in medium containing $25 \mathrm{mM}$ glucose for 48 h. All cell experiments were repeated three times.

\section{Statistical Analysis}

Statistical analyses were evaluated using GraphPad Prism 7.0 software (GraphPad Software, La Jolla, USA). All data are expressed as the mean \pm standard deviation. Intergroup data were compared by one-way ANOVA. A value of $\mathrm{P}<0.05$ was considered statistically significant.

\section{Results}

Yes-Associated Protein (YAP) Expression Increased in Rats with High Glucose

The protein expression of YAP was detected at different times. Immunohistochemical staining was used to measure the protein expression of YAP in vivo. The results showed that high glucose significantly increased YAP protein expression in the 4-week group, 12-week group and 16-week group compared with that in the control group (Figure $1 \mathrm{~A}, 1 \mathrm{E} ; \mathrm{P}<0.05$ ). YAP protein expression increased gradually over the 16 weeks.

\section{YAP Silencing Decreased YAP Expression in Rats with Diabetic Cardiomyopathy}

At the end of the experiment, YAP expression in the control group, DCM group, LV-SC-shRNA group and LV-YAPshRNA group was evaluated by immunohistochemical staining, PCR analysis and Western blot. The immunohistochemical staining results showed that YAP protein expression in the DCM group and LV-SC-shRNA group was significantly upregulated compared to that in the control group; however, this upregulation was significantly alleviated in the LV-YAPshRNA group (Figure $1 \mathrm{~B}, 1 \mathrm{~F} ; \mathrm{P}<0.05$ ). The results of YAP protein expression, as detected by Western blot, were consistent with the results of the immunohistochemical staining (Figure 1D, 1G P<0.05). Furthermore, the PCR results showed that YAP mRNA expression in the DCM group and LV-SCshRNA group was also significantly increased compared to that of the control group, and lentivirus-mediated YAP gene silencing significantly reduced myocardial YAP mRNA expression in the LV-YAP-shRNA group (Figure $1 \mathrm{C} \mathrm{P}<0.05$ ).

\section{YAP Silencing Decreased CTGF, Profilin- I and PAI-I Expression in Rats with Diabetic Cardiomyopathy}

Immunohistochemical staining was used to evaluate the protein expression of CTGF and Profilin-1. Our results 

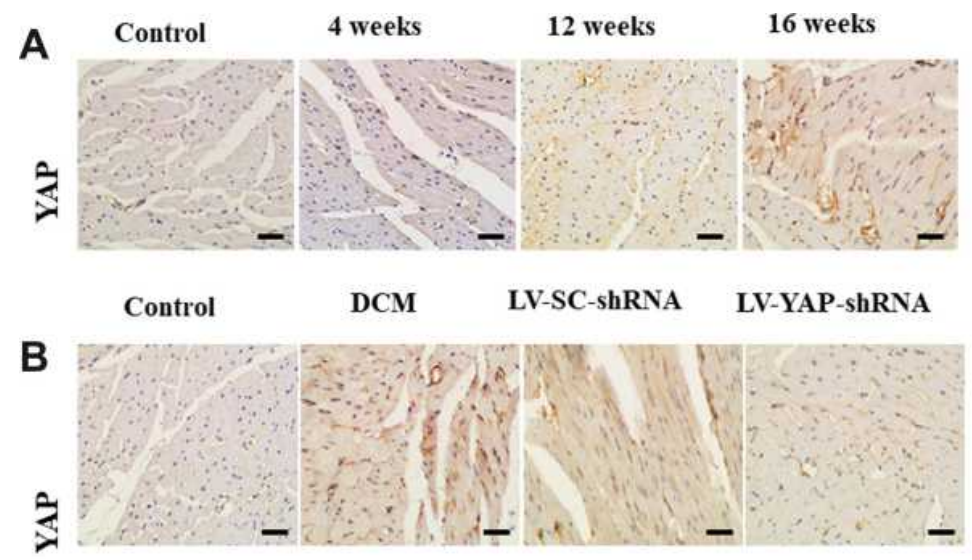

C

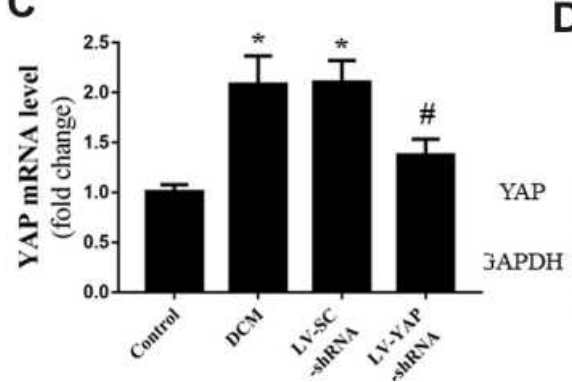

D

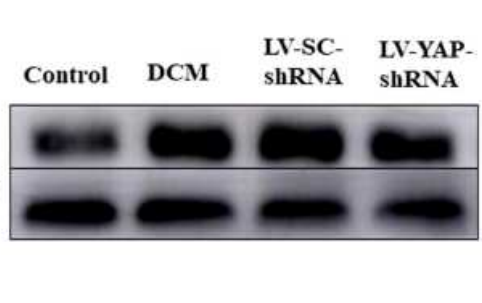

E

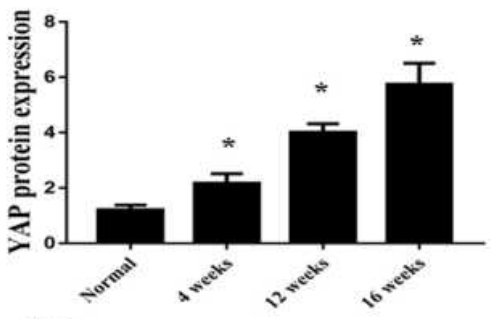

$\mathbf{F}$
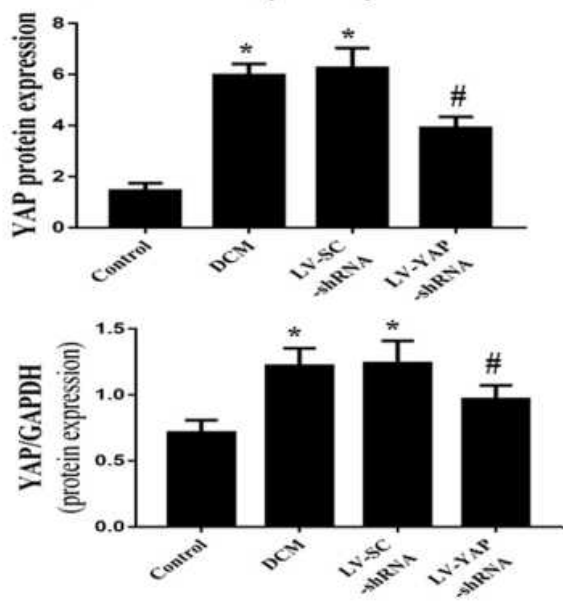

Figure I YAP expression in the myocardium in vivo $(n=6)$. (A) Representative immunohistochemical staining of YAP at different time points (scale bar=20 $\mu$ m). (B) Representative immunohistochemical staining of YAP in the 4 groups (scale bar $=20 \mu \mathrm{m}$ ). (C) YAP mRNA expression. (D) Western blot analysis of YAP protein expression. (E) Quantitative analysis of YAP protein expression shown in A. (F) Quantitative analysis of YAP protein expression shown in (B). (G) Quantitative analysis of YAP protein expression shown in (D). ${ }^{* P}<0.05$ versus the control group; ${ }^{\#} \mathrm{P}<0.05$ versus the LV-SC-shRNA group.

showed that CTGF protein expression in the DCM group and LV-SC-shRNA group was significantly increased compared to that of control group; however, CTGF protein expression was reduced in the LV-YAP-shRNA group compared to that of DCM and LV-SC-shRNA groups (Figure 2B and F). Moreover, the protein expression of Profilin-1 was much higher in the DCM and LV-SCshRNA groups than it was in control group, and it was reduced in the LV-YAP-shRNA group comparedto that of DCM and LV-SC-shRNA groups (Figure 2). Similarly, the protein expression of PAI-1 was much higher in the DCM and LV-SC-shRNA groups than it was in control group, and it was reduced in the LV-YAP-shRNA group compared to that of the DCM and LV-SC-shRNA groups. (Figure $2 \mathrm{D}$ and $\mathrm{G}$ ).

\section{YAP Silencing Decreased the Accumulation of Myocardial Interstitial Fibrosis in Rats with Diabetic Cardiomyopathy}

At the end of the experiment, myocyte hypertrophy was statistically lower in the LV-YAP-shRNA group than that in
LV-SC-shRNA group and DCM group (Figure 2A, E). The immunohistochemical staining results showed that the protein expression of collagen I, collagen III and PAI-1 was increased in the DCM group and LV-SC-shRNA group compared to the control group; however, the protein expression of collagen I, collagen III and PAI-1 was much lower in the LV-YAPshRNA group than it was in the DCM group and LV-SCshRNA group (Figure 2D and H, Figure 3A and B, F and G). Similarly, PCR results showed that collagen I, collagen III and PAI-1 mRNA expression in the DCM group and LV-SCshRNA group were also significantly increased compared to that of the control group, and their expression was significantly reduced in the LV-YAP-shRNA group compared to that of the DCM group and LV-SC-shRNA group (Figure 3C-E).

\section{YAP Silencing Improved Cardiac Function in Rats with Diabetic Cardiomyopathy}

At the end of the experiment, echocardiography was performed to evaluate cardiac function in the control group, DCM group, LV-SC-shRNA group and LV-YAP-shRNA group. The results showed that the left ventricular ejection fraction (LVEF) was much lower in the DCM group and LV- 

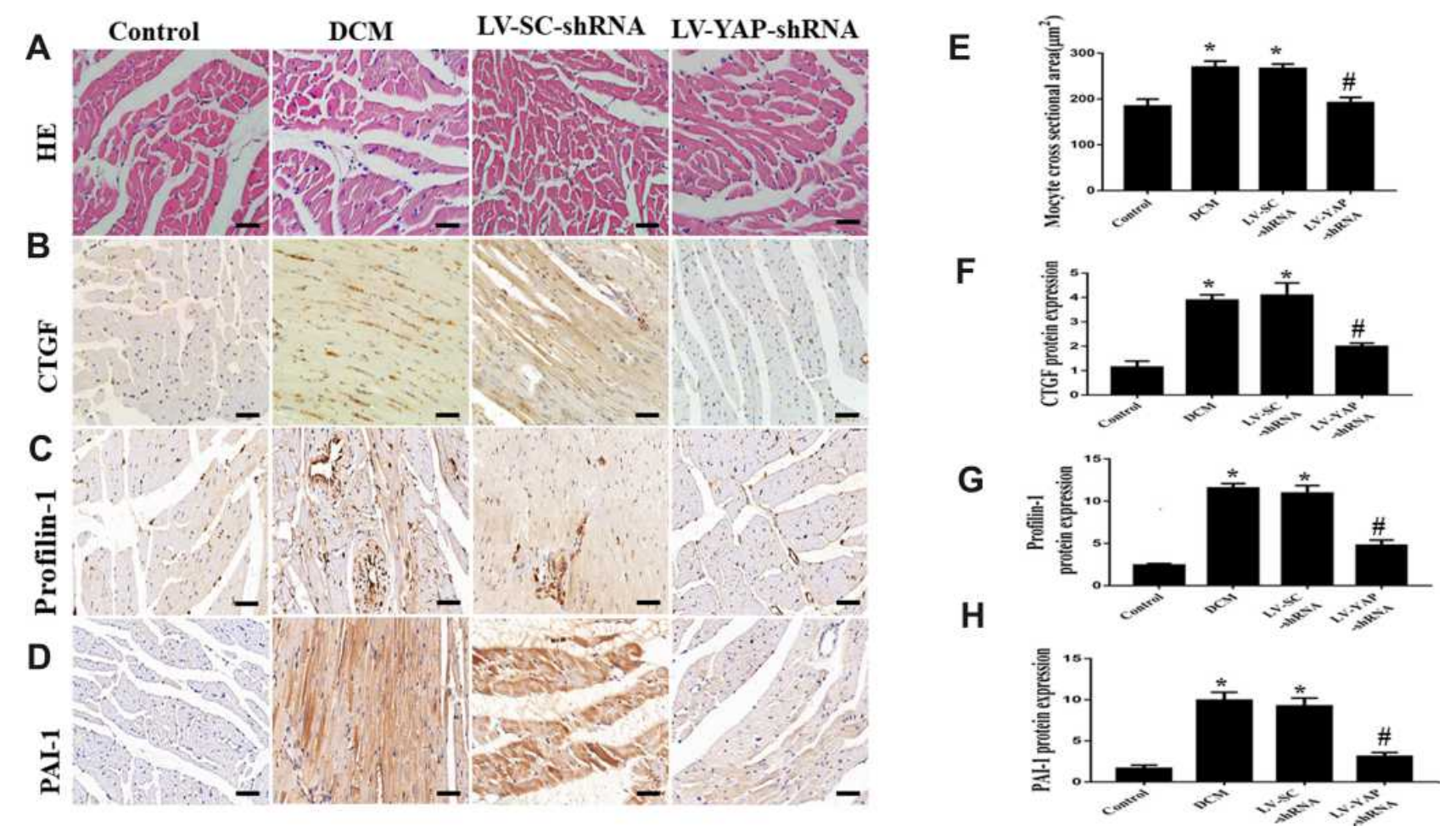

Figure 2 HE staining, CTGF, Profilin-I, and PAI-I protein expression in the myocardium in vivo $(n=6)$. (A-D) Representative HE staining and immunohistochemical staining of CTGF, Profilin-I and PAI-I in the 4 groups (scale bar $=20 \mu \mathrm{m}$ ). (E-H) Quantitative analysis of CTGF, Profilin-I, and PAI-I protein expression. $* \mathrm{P}<0.05$ versus control group; ${ }^{\#} \mathrm{P}<0.01$ versus LV-SC-shRNA group.
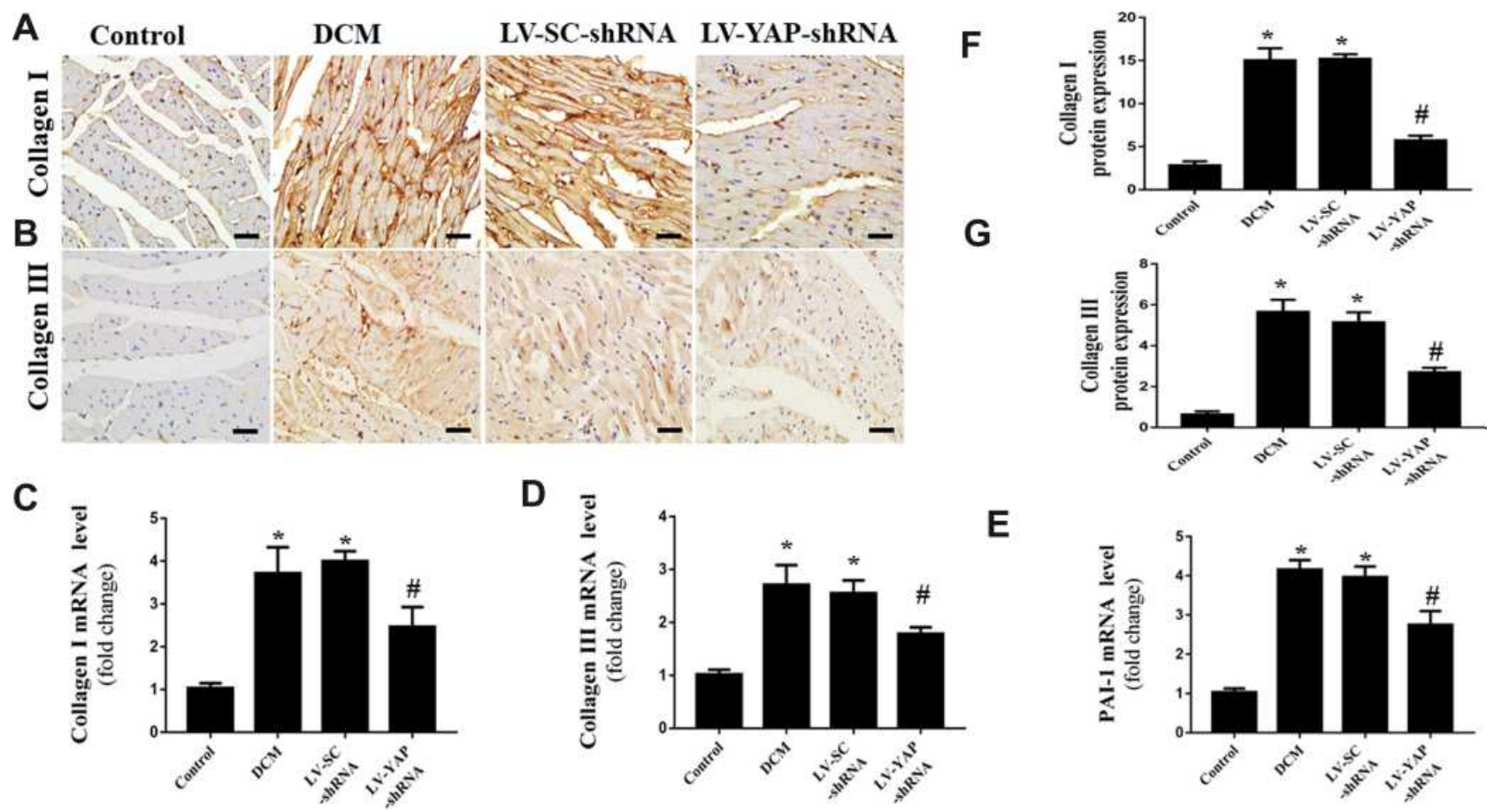

Figure 3 Collagen expression in the myocardium in vivo $(n=6)$. (A and B) Representative immunohistochemical staining of collagen I and collagen III in the 4 groups (scale bar $=20 \mu \mathrm{m}$ ). (C-E) Collagen I, collagen III and PAI-I mRNA expression in the 4 groups. (F and $\mathbf{G})$ Quantitative analysis of collagen I and collagen III protein expression shown in $(\mathbf{A})$ and $(\mathbf{B}) .{ }^{*} \mathrm{P}<0.01$ versus the control group; ${ }^{*} \mathrm{P}<0.01$ versus the LV-SC-shRNA group. 
A

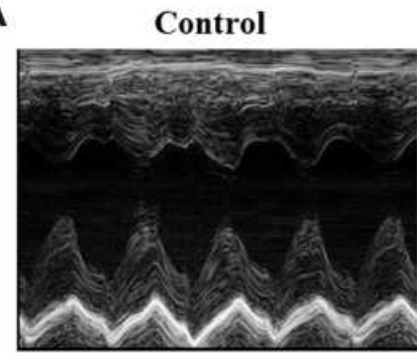

B

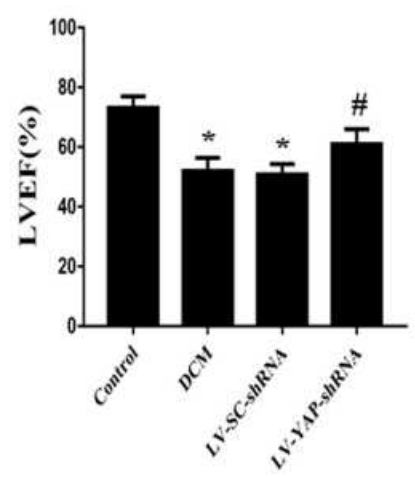

DCM

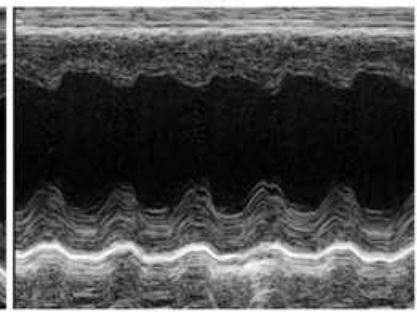

C

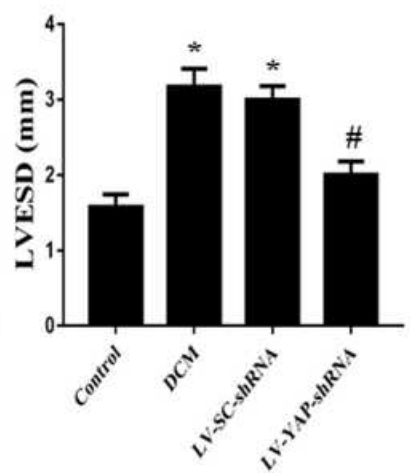

LV-SC-shRNA

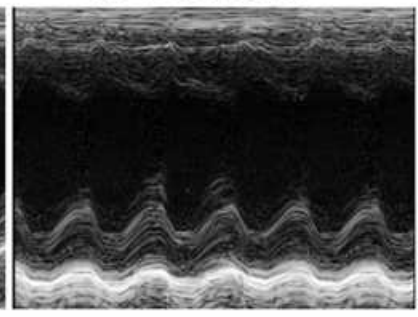

D

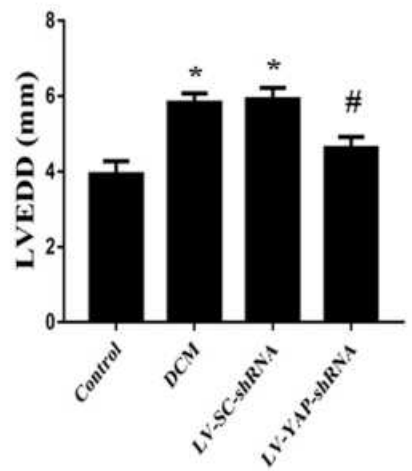

LV-YAP-shRNA

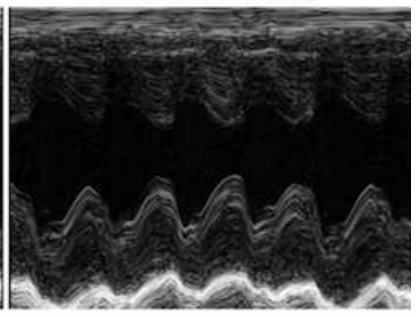

E

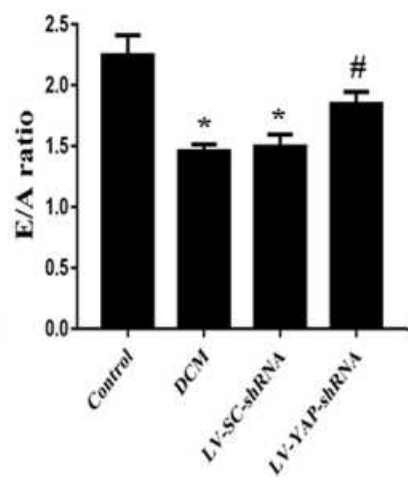

Figure 4(A) Cardiac function data in four groups of rats. M-mode echocardiograms in the 4 groups ( $n=5)$. (B) Indicated left ventricular ejection fraction (LVEF) in the 4 groups. (C) Left ventricular end systolic dimension (LVESD) in the 4 groups. (D) Left ventricular end diastolic diameter (LVEDD) in the 4 groups. (E) Ratio of peak early to late diastolic filling velocity (E/A ratio) respective levels in the 4 groups. ${ }^{*} \mathrm{P}<0.05$ versus the control group; ${ }^{\#} \mathrm{P}<0.05$ versus the LV-SC-shRNA group.

SC-shRNA group than it was in the control group; however, the LVEF in the LV-YAP-shRNA group was increased compared to that in the DCM group and LV-SC-shRNA group (Figure 4B). Left ventricular end-systolic diameters (LVESD) and left ventricular end-diastolic diameters (LVEDD) were markedly increased in the DCM group and LV-SC-shRNA group compared with the control group, which was lower in the LV-YAP-shRNA group than in the DCM group and LV-SC -shRNA group (Figure 4C and D). Meanwhile, accompanying the decrease in systolic function, cardiac diastolic function was also evaluated, and the results showed that the E/A ratio was higher in the LV-YAP-shRNA group than it was in the DCM group and LV-SC-shRNA group (Figure 4E).

\section{YAP Silencing Significantly Alleviated High Glucose-Induced CTGF and Fibronectin Protein Expression in Neonatal CFs via YAP in vitro}

First, we observed the protein expression of YAP at various time points under high glucose stimulation. The results showed that high glucose enhanced YAP protein expression in vitro and significantly increased YAP protein expression at $48 \mathrm{~h}$ (Figure 5A, C).
Second, we observed the effect of YAP short hairpin RNA silencing on YAP expression. In the present study, silencing YAP gene expression was achieved by short hairpin RNA (shRNA) technology. The Western blot results showed that the protein levels of YAP in the LV-YAP-shRNA group were lower than they were in the LV-SC-shRNA group (Figure 5B, E). Similarly, PCR results also showed that the mRNA expression of YAP in the LV-YAP-shRNA group was lower than it was in the LV-SC-shRNA group (Figure 5D).

Third, we observed the protein expression of CTGF and fibronectin in the LV-YAP-shRNA and LV-SC-shRNA groups with or without high glucose stimulation.

As the Western blot results revealed, the protein expression of CTGF and fibronectin in the LV-YAP-shRNA group was lower than that of the LV-SC-shRNA group. Furthermore, silencing of YAP significantly alleviated the high glucoseinduced increase in CTGF and fibronectin protein expression (Figure 5B, F, G).

\section{YAP Activated CTGF Through Interaction with TEADs in Neonatal CFs in vitro}

First, coimmunoprecipitation assays were performed to demonstrate the binding of YAP and TEADs. The Co-IP 

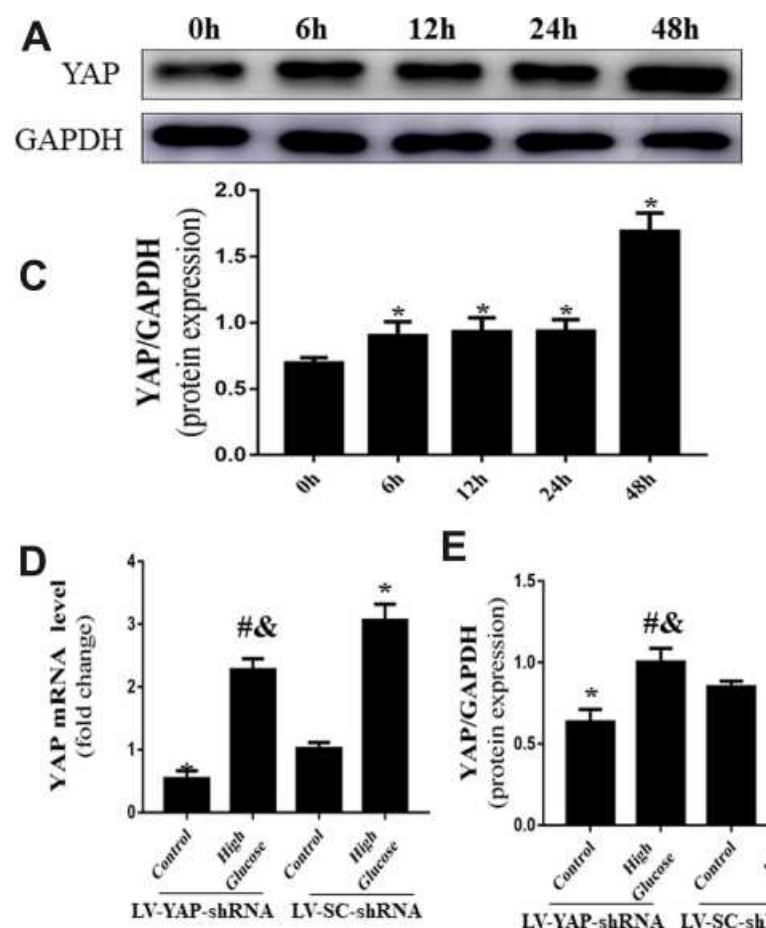

E

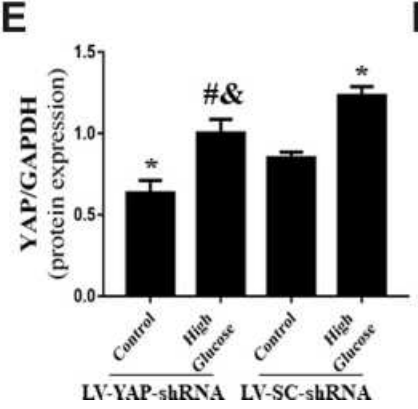

B LV-YAP-shRNA LV-SC-shRNA

GAPDH

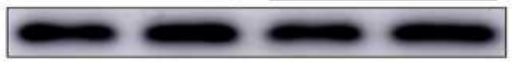

YAP

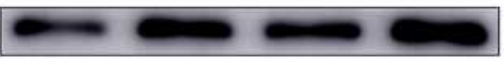

CTGF

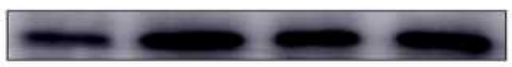

Fibronectin

High Glucose

$(-)$

$(+)$

$(-)$

$(+)$

$\mathbf{F}$

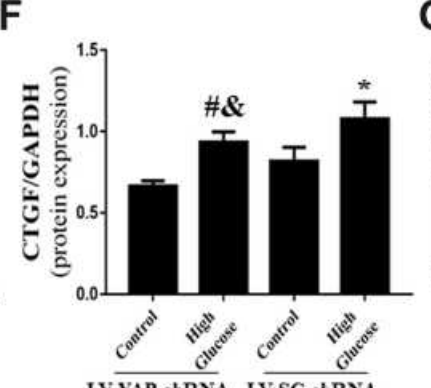

G

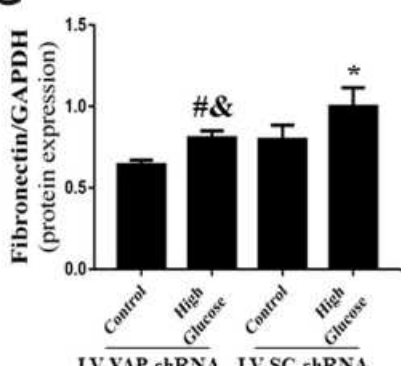

Figure 5 YAP expression and fibrosis levels in $C F s$ in vitro $(n=5)$. (A) Western blot analysis of YAP protein expression stimulated by high glucose $(25 \mathrm{mM})$ at different time points. (B) Western blot analysis of YAP, CTGF and fibronectin protein expression in CFs transfected with YAP-shRNA or scramble-shRNA. (C) Quantitative analysis of YAP protein expression shown in (A). (D) YAP mRNA expression in CFs transfected with YAP-shRNA or Scramble-shRNA. (E-G) Quantitative analysis of YAP, CTGF and fibronectin protein expression shown in (B). ${ }^{*} \mathrm{P}<0.05$ versus the control group transfected with Scramble-shRNA; ${ }^{\#} \mathrm{P}<0.05$ versus the high glucose group transfected with Scramble-shRNA; ${ }^{\&} \mathrm{P}<0.05$ versus the control group transfected with YAP-shRNA.

$\mathbf{A}_{\text {TEAD }}$

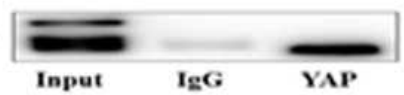

C
B

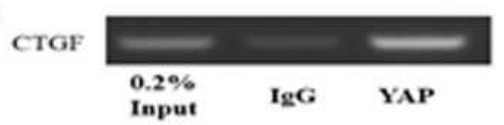

-186 TGCCAGCTTTTCAGACGGAGGAATGTGGAGTGTCAAGGG

-146 GTCAGGATCAATCCGGTGTGAGTtGATGAGGCAGGAAGGT

-106 GGGGAGGAATGCGAGGAATGTCCCTGTTTGTGTAGGACTC

-66 CATTCAGTTCTTTGGCGAGCCGGCCGCCCGGAGCGTATAA

-26 AAGCCAGCGCCACCCGCCCAGTCTCA(transcription initiation)

Figure 6 The Co-IP and Ch-IP in neonatal cardiac fibroblasts (CFs) in vitro. (A) The Co-IP results confirmed the interaction between YAP and TEAD in CFs. (B) The Ch-IP results showed YAP activates the CTGF promoter. (C) The amplified fragment of CTGF promoter contains a TEADs motif.

results showed that YAP coimmunoprecipitated with TEADs; IgG served as a negative control (Figure 6A).

Second, chromatin immunoprecipitation assays were performed to evaluate the activation of the CTGF promoter by YAP. The results were assessed by agarose electrophoresis and indicated that YAP activated the CTGF promoter through TEAD (Figure 6B). Five pairs of primers were designed for the Ch-IP assay based on the promoter sequence of CTGF in rats; only the fragment corresponding to one primer (the fifth primer) was amplified; the corresponding fragment is listed, and the corresponding fragment contains a TEAD motif (Figure 6C).

\section{Body Weight, Heart Weight, Blood Glucose and Blood Pressure Levels}

At the end of four weeks, Body weight and heart weight were significantly lower in DCM group, LV-SC-shRNA group and LV-YAP-shRNA group than that in Control group, whereas blood glucose level was statistically higher in DCM group, LV-SC-shRNA group and LV-YAP- 
Table I The Basic Characteristics of the 4 Groups

\begin{tabular}{|l|l|l|l|l|}
\hline Characteristics & Control & DCM & LV-SC-shRNA & LV-YAP-shRNA \\
\hline Weight(g) & $493.61 \pm 13.45$ & $330.99 \pm 19.28^{*}$ & $323.36 \pm 31.06^{*}$ & $381.51 \pm 18.47^{* \#}$ \\
Heart weight(g) & $1.70 \pm 0.11$ & $1.24 \pm 0.08^{*}$ & $1.30 \pm 0.07^{*}$ & $1.38 \pm 0.06^{*}$ \\
Blood Glucose (mmol/L) & $6.80 \pm 0.18$ & $27.96 \pm 1.39 *$ & $28.02 \pm 1.33^{*}$ & $26.20 \pm 1.5 I^{*}$ \\
Systolic Blood Pressure (mmHg) & $122.20 \pm 3.03$ & $122.25 \pm 2.38$ & $123.00 \pm 5.93$ & $123.75 \pm 5.26$ \\
Diastolic Blood Pressure (mmHg) & $90.10 \pm 2.11$ & $92.58 \pm 2.92$ & $91.25 \pm 2.57$ & $91.45 \pm 4.70$ \\
\hline
\end{tabular}

Notes: ${ }^{*} \mathrm{P}<0.01$ vs Control group; ${ }^{\#} \mathrm{P}<0.05$ vs LV-SC-shRNA group.

shRNA group than that in Control group. Systolic and diastolic blood pressure were not statistically different among DCM group, LV-SC-shRNA group, LV-YAPshRNA group and Control group (Table 1).

\section{Discussion}

Diabetic cardiomyopathy (DCM), which is characterized by structural and functional alterations, is a multifactorial disease resulting from diabetes mellitus that increases mortality in diabetic patients. Currently, there is still a lack of feasible therapeutic approaches for DCM. ${ }^{17}$ YAP is involved in diabetes and has been found in many studies. Previous studies showed that YAP protein expression increased in diabetic mice and played a crucial role in diabetic kidney treatment. Additionally, knocking down YAP expression with a specific siRNA could mediate renal epithelial injury in diabetes in response to high glucose or exogenous EGF. ${ }^{15}$ Moreover, recent studies have shown that YAP is reported to be closely related to cardiovascular disease. It has been reported that YAP is upregulated in human ischemic heart failure. Study found that inhibition of Hippo pathway may have a therapeutic benefit for patients with ischemic heart failure. ${ }^{18}$ Moreover, study showed that YAP and TAZ mechanotransduction mediates the pathological effects of disturbed blood flow on atherogenesis, correlating with key pathogenic events in atherosclerosis, such as endothelial thickening and recruitment of monocytes, which eventually turn into plaque-forming foam cells. ${ }^{11}$ In this study, we showed that the expression of YAP was increased in vivo and in vitro, suggesting that YAP is closely related to DCM. Then, we used a YAP shRNA to inhibit YAP expression in a DCM rat model and in high glucose-stimulated CFs to observe the role of YAP in the pathological progression of DCM.

Diabetic cardiomyopathy is an important factor in the morbidity and mortality of diabetic patients by causing heart failure. ${ }^{19}$ In pathological features of DCM, endoplasmic reticulum stress-induced autophagy, apoptosis and myocardial fibrosis play an important role. ${ }^{20,21}$ Myocardial fibrosis appears to be an important contributor to $\mathrm{DCM}^{22}$ The development of cardiac fibrosis is mainly manifested by abnormal proliferation of cardiac fibroblasts and deposition of extracellular matrix (ECM), which accelerates the pathological process of diabetic cardiomyopathy and adversely affects the function of the heart. ${ }^{23}$ Furthermore, cardiac fibrosis is associated with increased expression of transforming growth factor-beta (TGF- $\beta$ ), plasminogen activator inhibitor-1 (PAI-1), collagen and fibronectin. $^{24}$ In Ang II-stimulated cardiac fibrosis, YAP can be activated by Ang II to further affect cardiac fibrosis by regulating collagen expression, and inhibition of YAP by YAP inhibitor, verteporfin, attenuates Ang II-stimulated expression of collagen I. ${ }^{25}$ Furthermore, that report showed that YAP and TAZ are related mechanosensory proteins that bind to Smad transcription factors, which are the canonical mediators of profibrotic TGF- $\beta$ responses. YAP knockdown or treatment with verteporfin reduced YAP levels and decreased the total cellular levels of Smad2/3 after TGF- $\beta$ stimulation and attenuated fibrosis. ${ }^{26}$ Therefore, we hypothesized that YAP is associated with cardiac fibrosis in DCM and that YAP knockdown may have a favorable effect on DCM. In the present study, our result showed that YAP knockdown can alleviate cardiac fibrosis in the DCM rat model by reducing the expression of PAI-1, collagen I, collagen III, CTGF and profilin-1. YAP knockdown also reduced the expression of CTGF and fibronectin in CFs. In addition, the body weight and heart weight were significantly lower in the DCM, LVSC-shRNA and LV-YAP-shRNA groups than in the control group. The present study revealed that YAP played an important role in the pathological process of diabetic cardiomyopathy and that downregulating the expression of YAP may provide a novel therapeutic target for DCM.

Our study identified new aspects of YAP in the pathological progression of diabetic cardiomyopathy (DCM). In 
this study, our results showed that high glucose stimulation significantly increased the expression of YAP in primary rat CFs at $48 \mathrm{~h}$, and YAP was increased in the DCM rat model as early as week 4 and remained elevated through week 16. In the present study, YAP shRNA interference was used to inhibit YAP. In vivo, YAP silencing improved myocardial fibrosis in the DCM rat model. In vitro, YAP silencing also decreased the expression of CTGF and fibronectin in CFs stimulated by high glucose. DCM is often associated with left ventricular hypertrophy and reduced diastolic function, and it ultimately leads to heart dysfunction. ${ }^{3}$ In the present study, our results showed that cardiac systolic function and diastolic function were improved in the DCM rat model treated with a YAP shRNA, indicating that YAP silencing improved cardiac function in the DCM rat model. Therefore, YAP shRNAmediated interference could be used to achieve a therapeutic effect in cardiac function for diabetic cardiomyopathy, the mechanism may related to the decreased myocardial fibrosis.

The putative mechanisms underlying YAP promoting DCM fibrosis may be related to connective tissue growth factor (CTGF). CTGF, which is a cysteine-rich mitogenic peptide secreted by fibroblasts after activation with TGF- $\beta$, may serve as a more specific target for selective intervention in processes involving connective tissue formation during wound repair or fibrotic disorders. ${ }^{27}$ Previous studies have shown that the expression of CTGF is increased in a liver-specific YAP overexpression transgenic mouse model, and YAP regulates liver differentiation-dependent gene expression by influencing CTGF expression. ${ }^{28}$ In addition, CTGF has been identified as a direct YAP target gene and is important for cell growth. Recent studies have established that TEAD family transcription factors are essential in mediating YAP-dependent gene expression. TEAD is also required for YAP-induced cell growth, oncogenic transformation, and the epithelialmesenchymal transition. ${ }^{29}$ However, whether TEAD is required for YAP function and the mechanism by which YAP and TEAD mediate fibrosis in diabetic cardiomyopathy is still unclear. Therefore, we hypothesized that YAP may mediate CTGF by binding TEAD to aggravate fibrosis in diabetic cardiomyopathy. Herein, we used CFs to verify the relationship between YAP and CTGF. The protein expression of CTGF was decreased in CFs via YAP shRNA-mediated interference. Co-IP experiments showed binding between YAP and TEAD. Notably, based on the Ch-IP assays, the current work has identified that YAP is related to CTGF in a DCM rat model. In the Ch-IP assays, 5 pairs of primers were designed based on the promoter sequence of CTGF in rats, and only the fragment corresponding to one primer was amplified. We found that the corresponding fragment contained a TEAD motif. The results indicated that there is interaction between TEAD and YAP and it is possible that YAP activate CTGF promoter relying on the highlighted TEAD motif and further aggravate fibrosis in diabetic cardiomyopathy.

In conclusion, the results of the present study suggest that diabetes increases YAP expression in the myocardium. Then, YAP further promotes the molecular mechanism of myocardial fibrosis by regulating the gene expression of CTGF in diabetic cardiomyopathy, which can be prevented by YAP silencing. YAP gene therapy has a significant therapeutic impact on the pathological progression of diabetic cardiomyopathy, obviously attenuating myocardial fibrosis and improving cardiac function in diabetic rats. Therefore, our data may reveal a new potential treatment target for diabetic cardiomyopathy.

\section{Data Sharing Statement}

Data supporting the findings can be obtained by contacting the corresponding authors.

\section{Compliance with Ethical Standards}

All experiments were approved by the Animal Care and Use Committee of Shandong University, and all animal experiments were performed in accordance with the National Institutes of Health Guidelines for the Care and Use of Laboratory Animals. All efforts were made to minimize animal suffering.

\section{Acknowledgments}

Maomao $\mathrm{Hu}$, Han Wang and Shengnan Li are co-first authors for this study.

\section{Funding}

This study was supported by National Natural Science Foundation of China (No. 81870283, 81570729, 82070382) and Program of Taishan Scholars Programme (No. ts 20190979).

\section{Disclosure}

The authors declare that they have no conflicts of interest with the contents of this article. 


\section{References}

1. Huxley RR, Peters SAE, Mishra GD, Woodward M. Risk of all-cause mortality and vascular events in women versus men with type 1 diabetes: a systematic review and meta-analysis. Lancet Diabetes Endocrinol. 2015;3:198-206. doi:10.1016/S2213-8587(14)70248-7

2. Garcia MJ, McNamara PM, Gordon T, Kannel WB. Morbidity and mortality in diabetics in the Framingham population: sixteen year follow-up study. Diabetes. 1974;23(2):105-111. doi:10.2337/ diab.23.2.105

3. Mishra PK, Ying W, Nandi SS, Bandyopadhyay GK, Patel KK, Mahata SK. Diabetic cardiomyopathy: an immunometabolic perspective. Front Endocrinol. 2017;8:72. doi:10.3389/ fendo.2017.00072

4. Arow M, Waldman M, Yadin D, et al. Sodium-glucose cotransporter 2 inhibitor dapagliflozin attenuates diabetic cardiomyopathy Cardiovasc Diabetol. 2020;19:7. doi:10.1186/s12933-019-0980-4

5. Suzuki H, Kayama Y, Sakamoto M, et al. Arachidonate 12/15-lipoxygenaseinduced inflammation and oxidative stress are involved in the development of diabetic cardiomyopathy. Diabetes. 2015;64 (2):618-630. doi:10.2337/db13-1896

6. Shah MS, Brownlee M. Molecular and cellular mechanisms of cardiovascular disorders in diabetes. Circ Res. 2016;118(11):1808-1829. doi:10.1161/CIRCRESAHA.116.306923

7. Russo I, Frangogiannis NG. Diabetes-associated cardiac fibrosis: cellular effectors, molecular mechanisms and therapeutic opportunities. J Mol Cell Cardiol. 2016;90:84-93. doi:10.1016/j. yjmcc.2015.12.011

8. Sudol M. Yes-associated protein (YAP65) is a proline-rich phosphoprotein that binds to the $\mathrm{SH} 3$ domain of the yes proto-oncogene product. Oncogene. 1994;9(8):2145-2152.

9. Badouel C, Garg A, McNeill H. Herding hippos: regulating growth in flies and man. Curr Opin Cell Biol. 2009;21(6):837-843. doi:10.1016/j.ceb.2009.09.010

10. Halder G, Johnson RL. Hippo signaling: growth control and beyond. Development. 2011;138(1):9-22. doi:10.1242/dev.045500

11. Panciera T, Azzolin L, Cordenonsi M, Piccolo S. Mechanobiology of YAP and TAZ in physiology and disease. Nat Rev Mol Cell Biol. 2017;18(12):758-770. doi:10.1038/nrm.2017.87

12. Bertero T, Cottrill KA, Lu Y, et al. Matrix remodeling promotes pulmonary hypertension through feedback mechanoactivation of the YAP/TAZ-miR-130/301 circuit. Cell Rep. 2015;13(5):1016-1032. doi:10.1016/j.celrep.2015.09.049

13. Han Z, Yu Y, Cai B, et al. YAP/TEAD3 signal mediates cardiac lineage commitment of human-induced pluripotent stem cells J Cell Physiol. 2020;235(3):2753-2760. doi:10.1002/jcp.29179

14. Wang Y, Hu G, Liu F, et al. Deletion of yes-associated protein (YAP) specifically in cardiac and vascular smooth muscle cells reveals a crucial role for YAP in mouse cardiovascular development. Circ Res. 2014;114(6):957-965. doi:10.1161/CIRCRESAHA.114.303411

15. Chen J, Harris RC. Interaction of the EGF receptor and the hippo pathway in the diabetic kidney. $J$ Am Soc Nephrol. 2015;27(6):1689. doi:10.1681/ASN.2015040415
16. Wang $\mathrm{X}, \mathrm{Hu} \mathrm{G}$, Gao X, et al. The induction of yes-associated protein expression after arterial injury is crucial for smooth muscle phenotypic modulation and neointima formation. Arterioscler Thromb Vasc Biol. 2012;32(11):2662-2669. doi:10.1161/ATVBAHA.112.254730

17. Tian J, Zhao Y, Liu Y, et al. Roles and mechanisms of herbal medicine for diabetic cardiomyopathy: current status and perspective. Oxid Med Cell Longev. 2017;2017:8214541. doi:10.1155/2017/8214541

18. Tong M, Saito T, Zhai P, et al. Mitophagy is essential for maintaining cardiac function during high fat diet-induced diabetic cardiomyopathy. Circ Res. 2019;124(9):1360-1371. doi:10.1161/ CIRCRESAHA.118.314607

19. Leach JP, Heallen T, Zhang M, et al. Hippo pathway deficiency reverses systolic heart failure after infarction. Nature. 2017;550 (7675):260-264. doi:10.1038/nature24045

20. Yang L, Zhao D, Ren J, Yang J. Endoplasmic reticulum stress and protein quality control in diabetic cardiomyopathy. Biochim Biophys Acta. 2015;1852(2):209-218. doi:10.1016/j.bbadis.2014.05.006

21. Pei Z, Deng Q, Babcock SA, He EY, Ren J, Zhang Y. Inhibition of advanced glycation endproduct (AGE) rescues against streptozotocin-induced diabetic cardiomyopathy: role of autophagy and ER stress. Toxicol Lett. 2018;284:10-20. doi:10.1016/j. toxlet.2017.11.018

22. Armstrong AC, Ambale-Venkatesh B, Turkbey E, et al. Association of cardiovascular risk factors and myocardial fibrosis with early cardiac dysfunction in type 1 diabetes: the diabetes control and complications Trial/Epidemiology of Diabetes Interventions and Complications Study. Diabetes Care. 2017;40(3):405-411. doi:10.2337/dc16-1889

23. Villalobos E, Criollo A, Schiattarella GG, et al. Fibroblast primary cilia are required for cardiac fibrosis. Circulation. 2019;139 (20):2342-2357. doi:10.1161/CIRCULATIONAHA.117.028752

24. Zhou G, Wu J, Gu C, et al. Prorenin independently causes hypertension and renal and cardiac fibrosis in cypla1-prorenin transgenic rats. Clin Sci. 2018;132(12):1345-1363. doi:10.1042/CS20171659

25. Harikrishnan V, Titus AS, Cowling RT, Kailasam S. Collagen receptor cross-talk determines $\alpha$-smooth muscle actin-dependent collagen gene expression in angiotensin II-stimulated cardiac fibroblasts. J Biol Chem. 2019;294:19723-19739. doi:10.1074/jbc. RA119.009744

26. Szeto SG, Narimatsu M, Lu M, et al. YAP/TAZ are mechanoregulators of TGF-b-Smad signaling and renal fibrogenesis. J Am Soc Nephrol. 2016;27:3117-3128. doi:10.1681/ASN.2015050499

27. Grotendorst GR. Connective tissue growth factor: a mediator of TGF-beta action on fibroblasts. Cytokine Growth Factor Rev. 1997;8:171-179. doi:10.1016/S1359-6101(97)00010-5

28. Cheng J-C, Wang EY, Yi Y, et al. S1P stimulates proliferation by upregulating CTGF expression through S1PR2-mediated YAP activation. Mol Cancer Res. 2018;16(10):1543-1555. doi:10.1158/ 1541-7786.MCR-17-0681

29. Zhao B, Ye X, Yu J, et al. TEAD mediates YAP-dependent gene induction and growth control. Genes Dev. 2008;22:1962-1971. doi:10.1101/gad.1664408

Diabetes, Metabolic Syndrome and Obesity: Targets and Therapy

Dovepress

\section{Publish your work in this journal}

Diabetes, Metabolic Syndrome and Obesity: Targets and Therapy is an international, peer-reviewed open-access journal committed to the rapid publication of the latest laboratory and clinical findings in the fields of diabetes, metabolic syndrome and obesity research. Original research, review, case reports, hypothesis formation, expert opinion and commentaries are all considered for publication. The manuscript management system is completely online and includes a very quick and fair peer-review system, which is all easy to use. Visit http://www.dovepress.com/testimonials.php to read real quotes from published authors. 